\title{
Immunization strategies to elicit mucosal immune response of HIV Yinghui Shi*
}

Address: Qingdao CDC, Qingdao, Shandong, People Republic China, 266033

* Corresponding author

from 2006 International Meeting of The Institute of Human Virology

Baltimore, USA. 17-2I November, 2006

Published: 2I December 2006

Retrovirology 2006, 3(Suppl I):P68 doi:10.I I86/I742-4690-3-SI-P68

(C) 2006 Shi; licensee BioMed Central Ltd.

Since most human immunodeficiency virus (HIV) infection are initiated following mucosal to the virus, the anatomic containment or abortion of an HIV infection is likely to require vaccine-elicited cellular immune response in those mucosal sites. Studing vaccine-elicited mucosal immune responses have been problematic because of the difficulties associated with sampling T lymphocytes from those anatomic compartments. Herpes simplex virus type 1(HSV-1) infects a wide range of cells, including dendritics cells. Consequently, HSV-1 vectors may be capable of eliciting strong immune responses(Both celluar and humoral immune responses) to HIV.

To test this hypothesis, an HSV-1 amplicon plasmid encoding HIV-1 gp120 was constructed and murine immune response to helper virus-free amplicon were evaluated a sing intramuscular injection of HIV:gp120 amplicon (HSV:gp120)elicited Env-specific cellular and humoral immune response. The immune response to HSV:gp120 was durable, with robust cellular and longer humoral response. Finally, HSV: gp120 elicited significant Env-specific cellular immune response even in animals that had been previously infected with wild-type HSV-1 Taking together, these data strongly support the use of helper-free HSV-1 amplicon particles as vaccine delivery vectors to elicit mucosalimmune response of HIV. 